\title{
Regional Cooperation towards Effective Multi-Hazard Early Warnings in Asia
}

\author{
Kinkini Hemachandra ${ }^{\#}$, Richard Haigh ${ }^{\#}$, Dilanthi Amaratunga ${ }^{\#}$ \\ ${ }^{*}$ Global Disaster Resilience Centre, School of Art, Design and Architecture, University of Huddersfield, \\ Queensgate, Huddersfield, HD1 3DH, United Kingdom \\ E-mail: K.Hemachandra2@hud.ac.uk; R.Haigh@hud.ac.uk; D.Amaratunga@hud.ac.uk
}

\begin{abstract}
Due to the unprecedented rate of climate change, the number of coastal hazards have been significantly increased causing a large number of deaths and economic losses during the $21^{\text {st }}$ century. Hence, leading global initiatives emphasise the necessity of multi-hazard early warnings for effective disaster risk reduction and resilience across the world. Asia reported the largest share of deaths and economic losses from all type of natural hazards, showing gaps within their coping capacities and gaps in present preparedness measures. Multi-hazard early warnings play a significant role within present disaster risk reduction measures irrespective of disaster type. Regional cooperation in this effort could be further benefitted due to its ability of sharing knowledge and costs among member countries. Nevertheless, present situation of multi-hazard early warnings in Asia does not deliver its expected results due to many uncovered reasons. Hence, this paper is written with the objective of identifying enablers and barriers that affect effective multi-hazard early warnings in Asia. This paper is based on the findings of an initial stage of a project aimed at enhancing capacity building among Asian higher education institutions through capacity development programmes for an effective risk reduction and resilience mechanisms. Accordingly, the study conducted an online survey among experts in multi-hazard early warnings and found that, risk warnings, governance, awareness and education, preparedness culture and resources as the enablers for effective multi-hazard early warnings in Asia. The study also revealed that weak monitoring, weak preparedness and response capacities and weak regional and political support as the barriers for successful implementation.
\end{abstract}

Keywords — coastal hazards; Asia; multi-hazard early warnings; enablers; regional cooperation.

\section{INTRODUCTION}

Climate change has been unprecedentedly increased during the last century causing a large number of deaths and economic losses across the world [1]. Specifically, climate related disaster risks have been increased in coastal cities [2] because of rising sea level [3]. As a result, coastal communities have become frequently vulnerable in many types of coastal hazards during the last couple of decades [2, 4]. This is furthermore complicated by increasing population who live nearby coastal areas [5]. The number of people live nearby coastal regions is furthermore expected to be risen when compared to other regions [4].

Asia is more vulnerable to different type of coastal hazards. Recent tsunami incidents in Palu and Sulawesie, in Indonesia [6], Cyclone Nargis in Myanmar [7], the Indian Ocean Tsunami incident in 2004 which killed more than 230,000 people across 14 countries in Asia and the Pacific region [8] show the risk of increasing risk of coastal hazards in Asia.
In addition, coastal erosion, oil spills, wind storms, and flooding alike are other sources of major coatal hazards in Asia [4]. During 1985-2006, 57\% of affected people by coastal hazards were reported in Asia [9]. Furthermore, Asia reports the largest share of poor people living on the earth, making further complexities to these natural hazards. This is furthermore worsened in small islands and tropical developing countries where the coastal communities represent the poorest group in the society [10]. Thus, weak coping capacities make the system further vulnerable [2]. Increasing population [11], migration [12] and diverging socio-economic conditions generate further complexities when dealing with coastal hazards [4, 9] in Asia.

In order to reduce the impact of coastal hazards among coastal communities, global frameworks, policy makers and practitioners highlighted the importance of multi-hazard early warnings (MHEW) as an effective strategy to reduce disaster risks [13-15]. For example, the 7th target of the Sendai Framework for Disaster Risk Reduction (SFDRR) underlines the necessity of increasing availability of and access to MHEW, disaster risk information and assessments to people by 2030 [16]. Similarly, the Sustainable 
Development Goals (SDGs) recognizes the significance of global partnerships enabling poor and vulnerable countries, through provisioning of resources for effective risk reduction and resilience. Specifically, its 13th Goal emphasizes the necessity of enhancing human and institutional capacities in MHEW [17].

Hence, several mechanisms and initiatives have been established in Asia to address this urgent need in the region. For example, establishment of the Indian Ocean Tsunami Warning \& Mitigation System (IOTWMS) in 2011 [18, 19], setting up of 24 tsunami warning centres in the Indian Ocean, and expanding the number of deep ocean tsunameters for data sharing are some of the initiatives taken place in the region [20].

However, existing systems have not been completely able to reduce the risk of coastal hazards in Asia [21]. For an example, there are many instances where communication of early warnings did not reach the most vulnerable communities [5, 22]. Similarly, many other issues and weaknesses are found in terms of collecting and receiving warning messages, acquisition of real time data and lack of equipment in the region [23]. Hence, it is highlighted the necessity of developing platforms for knowledge sharing in the region as an effective way of capacity building [18]. According to Lumbroso, et al. [21] study, there is a urgent need to carefully identify and study indepth the exact reasons for such weaknesses in MHEW in Asia.

Hence, this project aims to fulfil this gap by investigating gaps and challenges for establishing an effective MHEW in the region limiting to the scope of capacity building among members from higher-education institutions (HEIs) in Asia. This paper is based on the findings of its second stage of the project "Capacity Building in Asia for Resilience Education (CABARET)" with following objectives:

(1) to explore present status of regional cooperation towards effective MHEW in Asia;

(2) to identify the enablers that affect effective functioning of MHEW in Asia and

(3) to identify the barriers that hinders their effective functioning of MHEW in Asia.

The rest of the paper is structured, by presenting materials and methods in Section II, results and discussion in Section III and presenting conclusions of the study in Section IV.

\section{MATERIAL AND METHODS}

The study conducted a detailed literature review to develop its initial conceptual framework and desigining data collection instruments. Table I presents the documents referred within this review.

After this documents review, a consultative process was conducted with 16 Asian experts representing Indonesia, Maldives, Myanmar, Philippines and Sri Lanka and 9 European experts representing Bulgaria, Latvia, Malta, Spain and the UK.
TABLE I

LIST OF DOCUMENTS REVIEWED

\begin{tabular}{|l|c|}
\hline \multicolumn{1}{|c|}{ Documnt category } & $\begin{array}{c}\text { Number of } \\
\text { documents }\end{array}$ \\
\hline Peer reviewed journal papers & 16 \\
\hline $\begin{array}{l}\text { Official reports published by the UN, UNDP } \\
\text { and governments }\end{array}$ & 16 \\
\hline Official websites & 10 \\
\hline Total & 42 \\
\hline
\end{tabular}

The initial literature review and experts opinions were used to identify 15 enablers that affect effective functioning of MHEW and presented a capacity analysis framework for MHEW for coastal community resilience [14].

Then, a questionnaire survey was conducted among experts in disaster risk reduction and resilience and MHEW systems in Asia. The questionnaire survey consisted with opened and closed ended questions. Some matrix questions were asked to rate their preferences on the given statements. They were in the forms of scale questions, ranking questions and dichotomous questions. During July- October 2017, an online survey was launched using the Survey Monkey instrument to understand and evaluate regional perspective in terms of MHEW in Asia.

The research design for the study was a mixed method which enables the collection and analysis of both qualitative and quantitative methods in a study [24]. The data were analysed descriptively and quantitatively using SPSS 23.0 version statistical software. 136 responses were fully completed out of 199 survey responses. The study conducted reliability test for questions using Cronbach's Alpha statistical method. The sampling adequacy of question was tested using KMO and Barletts' tests. Principal Component Analysis (PCA) was used to identify key categories of enablers and barriers which were identified through literature review.

\section{A. Respondents' profile}

Table II presents respondents' profile which shows diversity of the sample. The respondents from other category represents UNDP agents, international agencies which deals with capacity building in DRR and scientific societies.

TABLE II

RESPONDENTS' PROFILE

\begin{tabular}{|l|c|}
\hline Respondents' organization & Percentage (\%) \\
\hline Higher education institutions & 30 \\
\hline Emergency relief \& humanitarian (NGOs) & 29 \\
\hline Government & 26 \\
\hline Private sector & 6 \\
\hline Volunteer associations & 1 \\
\hline Media & 1 \\
\hline Other & 7 \\
\hline Total (136) & 100 \\
\hline
\end{tabular}

In addition, $70 \%$ of respondents have less than 10 years of working experience in disaster risk reduction (DRR) and early warning sectors. Out of, 53\% of respondents have 
more than 5 years of experience in disaster risk reduction field. There were $7 \%$ of respondents who have experience in the field for more than 20 years.

$23 \%$ of respondents engages in governance and organizational coordination whereas another $22 \%$ of respondents involve in emergency preparedness and response operations. 67 responents represents $17 \%$ of the sample involved in issuing warning messages. The lowest level of involvement among the respondents is in communication activities which accounted for $10 \%$.

\section{RESULTS AND DISCUSSION}

\section{A. Regional Cooperation}

First objective of the study is to explore present status of regional cooperation within MHEW in Asia based on survey results. Accordingly, the respondents were ask to express their level of agreement with given four statements. In order to assure the level of reliability, Cronbach's Alpha test was carried out and test scored 0.825, which confirms the reliability of the question. Reespondents were asked to mark their level of agreement based on an ordinal scale measurement. Measurements are ranked from strongly disagree to strongly agree where, strongly disagree $=1$, disagree $=2$, undecided $=3$, agree $=4$ and strongly agree $=5$.

According to Table III, 49\% agrees and 10\% strongly agrees that stakeholders confirm that key stakeholders are consulted when taking initiatives or actions in developing MHEW in Asia. Another $49 \%$ and $7 \%$ confirm that availability of inter-regional key stakeholder partnerships in Asia. In total, $47 \%$ agreed that there is a regionalism approach when developing MHEW in the region. However, $36 \%$ disagree with effectiveness of existing stakeholder partnerships for developing MHEW in Asia.

TABLE III

REGIONAL COOPERATION ON MHEW IN ASIA

\begin{tabular}{|l|c|c|c|c|c|}
\hline \multirow{2}{*}{ Area } & \multicolumn{5}{|c|}{ Preferences (\%) } \\
\cline { 2 - 6 } & $\mathbf{1}$ & $\mathbf{2}$ & $\mathbf{3}$ & $\mathbf{4}$ & $\mathbf{5}$ \\
\hline $\begin{array}{l}\text { Key stakeholders' consultation } \\
\text { when developing MHEW }\end{array}$ & 9 & 16 & 17 & 49 & 10 \\
\hline $\begin{array}{l}\text { Availability of key stakeholder } \\
\text { partnerships in Asia }\end{array}$ & 9 & 13 & 22 & 49 & 7 \\
\hline $\begin{array}{l}\text { Regionalism approach in } \\
\text { developing MHEW in Asia }\end{array}$ & 10 & 21 & 21 & 36 & 11 \\
\hline $\begin{array}{l}\text { Effective partnerships for } \\
\text { developing MHEW in Asia }\end{array}$ & 7 & 36 & 18 & 32 & 8 \\
\hline
\end{tabular}

Member countries in the Asia-Pacific region demand for a regional cooperation to share scientific knowledge \& applications, as well as to share costs when dealing with trans-boundary hazards [22]. Thus, Indian Ocean Consortium was established to support the development of national components of the Indian Ocean Tsunami Warning Monitoring System (IOTWMS), through coordination mechanisms among governments, preparing national plans for tsunami warnings and creating links between regional efforts [25].

In summary, there is a regionalism approach, in Asia. Nevertheless, existing stakeholder partnerships are still not effectively contributing to enhance coastal resilience in Asia. This gap clearly revealed by the survey needs to be addressed in Asia for an effective coastal resilience through MHEW through regional cooperation. For example, Severe Weather Forecasting Demonstration Project was introduced to fulfil the needs of MHEWs as a regional project to strengthen capacities among high risk and low capacity countries [22].

Despite the importance of regional cooperation for enhancing resilience among coastal communities, many challenges hinder their effective functioning. For example, uneven progress in early warning systems among member countries due to: high risk involvement, low capacities, different types of hazards and lack of implementation on the last mile warning systems [18]. In addition, unsatisfactory institutional arrangements [18], weak warning systems [26] and weak capacities [5] are prevalent in the Asia- Pacific region. For example, early warning messages were not disseminated among coastal communities in Indonesia, Thailand and Malaysia during the Indian Ocean Tsunami incident in 2004 [5]. Similarly, MHEW is isolated from policy and decision-making process in the region. Hence, it is required to establish appropriate level of stakeholder partnerships as well. Furthermore, dissemination of risk information among the most vulnerable communities, for an example, fishing communities, are limited [22]. In order to address these challenges, it is necessary to establish platforms for knowledge sharing in the region [18].

Thus survey further aimed to identify key areas to be further improved for an effective regional cooperation in Asia. Three statements were given with five ordinal scale measurements: very low $=1$, low $=2$, medium $=3$, high $=4$ and very high $=5$. Those three aspects have been identified through literature review as well as through the onsultative process with partners.

$42 \%$ considered that capacity development needs are in high priority when developing regional cooperation. Similarly, 44\% agreed that enhancing innovations in MHEW as a another priority. Another 52\% confirmed that training needs as another priority when establishing regional cooperation for effective MHEW in Asia.

In parallel, an open-ended question was raised to identify any other areas to be improved towards coastal resilience when establishing regional cooperation in Asia. Following areas were proposed by respondents to the survey;

- Integration of local people as stakeholders when establishing regional cooperation for the development of MHEW,

- Development of proper systems and mechanisms towards regional cooperation,

- Knowledge sharing and networking in the region,

- Use of ICT and computer modelling,

- Mainstream MHEW into development planning,

- Provisioning of emergency information systems for the public,

- Creation of links between government disaster management units and universities and

- Disaster education and awareness building. 
In this regard, the contribution of this project will be vital to establish and enhance capacities through knowledge generation and sharing at regional level.

\section{B. Enablers}

In addition to identified gaps within regional cooperation, there are many factors affecting effective functioning of MHEW. Based on literature review, the study identified 15 enablers that affect effective functioning of MHEW. They are; governance [27], training, awareness and education, community participation [22], level of involvement of local authorities [28], planning and preparedness [15], technical and scientific information and knowledge [18], risk information, resources and infrastructure, hazard warning [29], political recognition [26], stakeholder partnerships [30], mainstreaming disaster risk reduction and climate change adaptation into development planning [15], monitoring and forecasting [30] and communication [18].

Accordingly, objective 2 was achieved by giving 41 statements to cover identified 15 enablers. They were arranged as ordinal scale measurements with five measurement scales. The scales are: strongly disagree $=1$, disagree $=2$, undecided $=3$, agree $=4$ and strongly agree $=5$. Hence, respondents were asked to reveal their opinion about each factor for the enhancement of MHEW in Asia. Data was analysed using Principal Component Analysis (PCA) in order to identify major categories of factors.

Before conducting the test, the reliability of questions were measured. This is specifically important when there are more factors available within a question. The Cronbach's Alpha was 0.954 which indicates a higher reliability and internal consistency among factors. Similarly, KMO and Bartlett's tests were carried out to measure the sample adequacy of the tests.

After confirming both reliability and sample adequacy tests, PCA was carried out to identify key components among enablers tested in the survey. Based on results of the correlation values among all 41 factors, five components were identified. The identified components are based on variables that are most strongly correlated with each component. Table IV presents all five components and their correlation values within variables. The objective was to identify common pattern of many variables that were strongly agreed or agreed by respondents. Hence, PCA is suitable to understand underlying components that constituted for these responses.

The rule of thumb for PCA is to keep the principal components which have eigenvalues greater than 1. Lower variances are reported from variables with lower eigenvalue which are of little use. Principal components analysis is to redistribute the variance in the correlation matrix (using the method of eigenvalue decomposition) to redistribute variance to first components extracted. Higher correlation values demonstrate higher correlation between variables within a component.
Hence, Table IV has been improved to demonstrate the highest correlated variables within each component to identify and label for a meaningful data reduction strategy. All these variables are positively related to effectiveness of MHEW. For example, when risk based warnings increase, the effectiveness of MHEW increases. Accordingly, component 1; risk based waning, sharing knowledge, updates of hazards and risk maps are highly correlated within this component. Based on their similarity or common characteristics, Component 1 is named to represent the relationships among the highest correlated components. Accordingly, Component 1 is considered as a measure of risk warning. Literature furthermore confirmed that risk warning as a fundamental element in effective MHEW. For example, issue of early warning messages to people who are marginalized and not involved in the development process with adequate time is a key towards disaster risk reduction and resilience [18, 22, 26, 31].

The second component was named as governance since all the highest correlated values are related to institutions. The highest correlated value is reported from policy availability. Provision of capacity building, hazard monitoring feedback and innovations research based policy making are the next highest correlated elements in the table. All four elements are positively correlated to each other and in the same direction. This is because; governance helps in coordinating science with policy and practice for effective early warning system along with resource allocation [26]. Governance along with strong leadership, legal frameworks and institutions similarly enhance resilience capacities. Hence, governance and institutional arrangements are considered as fundamentals to the development and maintenance of effective early warning systems [30].

Third component is related to awareness and education. Hence, it was named as awareness and education. Accordingly, stakeholder awareness is highly important factor towards awareness and education. Any change in the third component will be related to a change in stakeholder awareness. Similarly, school and university awareness and media campaign also increase education and awareness as enablers. Education and awareness is considered by many scholars for effective functioning of MHEW [15]. According to UNESCO consolidated report, present level of awareness and education within the Indian Ocean member countries is not at a satisfactory level [23].

TABLE IV

ENABLERS FOR EFFECTIVE FUNCTIONING OF MHEW IN ASIA

\begin{tabular}{|l|c|c|c|c|c|}
\hline \multicolumn{1}{|c|}{ Enablers } & \multicolumn{3}{|c|}{ Components } & \multicolumn{1}{l|}{} \\
\hline & $\mathbf{1}$ & $\mathbf{2}$ & $\mathbf{3}$ & $\mathbf{4}$ & $\mathbf{5}$ \\
\hline Risk based warning & 0.820 & & & & \\
Updates of hazards & 0.814 & & & & \\
Sharing knowledge & 0.814 & & & & \\
Risk maps & 0.812 & & & & \\
\hline Policy availability & & 0.696 & & & \\
Capacity building & & 0.682 & & & \\
Monitoring feedback & & 0.678 & & & \\
Innovation research & & 0.667 & & & \\
\hline Stakeholder awareness & & & 0.640 & & \\
School university & & & 0.617 & & \\
$\quad$ awareness & & & & & \\
Media campaign & & & 0.607 & & \\
\hline
\end{tabular}




\begin{tabular}{|l|l|l|l|l|l|}
\hline Engagement in planning & & & & 0.459 & \\
Preparedness culture & & & & 0.441 & \\
\hline Financial sustainability & & & & & 0.325 \\
Resource availability & & & & & 0.325 \\
\hline
\end{tabular}

Fourth component was named as preparedness culture. Their engagement in planning and creating a preparedness culture is highly correlated as enablers for effective MHEW. These two elements are moving in the same direction. The importance of planning and preparedness have been identified in number of studies. For example, Alfieri, et al. [15] study highlights preparedness as a key for reducing adverse effects of natural disasters Furthermore, the SFDRR emphasise the importance of preparedness in its fourth priority [17]. However, it is evident that most preparedness measures are ad-hoc, fragmented and lack of proper coordination with other development plans in Asia [32].

The last component of the PCA, identified as Resources. Financial sustainability and resource availability are correlated each other and having an eigenvalue more than 1 . Effective early warnings ensure preparedness measures towards risk reduction and resilience [15, 30]. Adequate allocation of resources is considered as a key element for the smooth functioning of any system [30] However, this is not recognized by the survey as a significant component since its correlated values are lower than its required level.

In summary, out of 41 factors identified as enablers for an effective MHEW in Asia, five categories were formed as: Risk warning; Governance; Awareness and education; Preparedness culture and Resources.

\section{Barriers}

In order to achieve third objective of the study, a question was designed to identify the barriers that affect effective functioning of MHEW in Asia. They were raised as ordinal scale measurement questions with five different scales as: $1=$ strongly disagree; $2=$ disagree; $3=$ undecided; $4=$ agree and $5=$ strongly agree.

As the first requirement of conducting PCA, the study conducted a reliability test by calculating Cronbach's Alpha which was 0.926 . It complied with the standard rule of being more than 0.7 . The sample adequacy test also carried out and satisfied.

Based on PCA, three main categories were identified as barriers for effective functioning of MHEW in Asia. Based on the highest value of correlations among each variable, three categories were identified and named as: weak monitoring; weak preparedness and response capacities; and weak regional and political support.

TABLE V

BARRIERS FOR EFFECTIVE FUNCTIONING OF MHEW IN ASIA

\begin{tabular}{|c|c|c|c|}
\hline \multicolumn{4}{|c|}{ Categories } \\
\hline Variables & 1 & 2 & 3 \\
\hline Lack of responsive institution & 0.738 & & \\
\hline Lack of implementation & 0.727 & & \\
\hline Lack of effective use of EWS & 0.724 & & \\
\hline Lack of integrating early warning & & 0.580 & \\
\hline Lack of public education & & 0.578 & \\
\hline $\begin{array}{l}\text { Lack of integration of diversity of } \\
\text { livelihood choices }\end{array}$ & & 0.571 & \\
\hline Lack of regionalism approach & & & 0.370 \\
\hline Lack of political public appreciation & & & 0.348 \\
\hline
\end{tabular}

Monitoring will be weak when there is lack of responsive and innovative institutions for conducting research, education and implementation in the region. Lower the availability of these institutions, the effectiveness of MHEW cannot be achieved in Asia. Furthermore, lack of responsive institutions positively affects towards lack of implementation as well as the use of EW effectively in Asia. Literature too highlights that there are many aspects in the institutional arrangements for further development in Asia [32].

The second component identified by the PCA, is related to weak preparedness and response capacities within the region as a barrier towards effective MHEW. Lack of integration of early warnings towards multi-purpose monitoring do not save lives and donot support livelihoods. Lack of this integration, hence hinders response capacity. Similarly, this also highly related with lack of education and awareness at the regional level. Weak preparedness reduces both response capacity and community resilience. This is identified as one of the major barrier towards effective MHEW in the region. As stated earlier, preparedness culture is a key towards effective DRR and resilience [31].

The third component is related to weak regionalism approach and political support. Lack of collective actions for promoting MHEW is a barrier in the region. Similarly, weak regionalism drives to poor level of public and political appreciation for identifying vulnerabilities and their consequences in the region. Political leadership and commitment contribute to the success of any DRR initiatives [15] inclusive of EWS [26]. Thus, political commitment supports resource allocation and introduction of legal mechanisms. This has been progressed in Asia and the Pacific region. For example, the Government of the Philippines introduced a Zero Casualty policy after following the Typhoon Haiyan in 2013. It has shown a substantial reduction in terms of loss of lives and number of people affected from the Typhoon Koppu in 2015 [17].

\section{CONCLUSIONS}

Asia, as a single region, reported the highest number of coastal hazards due to chaninging climatic condition. Hence, effective MHEW with a strong regional cooperation is recommended as an effective strategy to reduce the risk of coastal hazards and resilience in Asia. Nevertheless, present level of regional cooperation for developing MHEW is suffering with many capacity gaps and challenges. The survey identified a strong need of developing capacities, engagement of innovative research and training needs as the most promonent areas for effective regional cooperation in Asia while ensuring integration of local people into MHEW development, mainstreaming MHEW into development planning, knowledge sharing and networking, use of developed methods for risk identification towards an effective MHEWS in Asia. In addition, for an effective MHEW, risk warning; governance; awareness and education; preparedness culture and resources are identified as the key enablers in Asia. Similarly, three major gaps/ challenges were identified as weak monitoring; weak preparedness and response capacities; and weak regional and political support towards effective functioning of MHEW in Asia. 
As highlighted earlier, capacity developments in Asia could contribute to enhance effectiveness of MHEW as well as to overcome existing barriers. Hence, this study contributes to address this timely important need towards coastal resilience in the region. After identifying this capacity gaps, the project has conducted its capacity development activities as the next stage of the project.

\section{ACKKNOWLEDGEMENT}

The European Commission support for the production of this publication does not constitute an endorsement of the contents which reflects the views only of the authors, and the Commission cannot be held responsible for any use which may be made of the information contained therein.

\section{REFERENCES}

[1] CRED - UNISDR, "Poverty \& Death: DISASTER MORTALITY " in "Mortality trends from major disasters from 1996 to 2015 " 2016.

[2] J. S. Setyono and R. K. Yuniartanti, "The challenges of disaster governance in an Indonesian multi-hazards city: a case of Semarang, Central Java," Procedia-Social and Behavioral Sciences, vol. 227, pp. 347-353, 2016.

[3] IPCC, "Summary for policymakers. In: Climate Change 2014: Impacts, Adaptation, and Vulnerability. Part A: Global and Sectoral Aspects. Contribution of Working Group II to the Fifth Assessment Report of the Intergovernmental Panel on Climate Change," Intergovernmental Panel on Climate Change, Cambridge University Press, Cambridge, United Kingdom and New York, NY, USA, 2014.

[4] Mark D Spalding, C. L. Susan Ruffo, and L. Z. H. Imen Meliane, Christine C Shepard, Michael W Beck, "The role of ecosystems in coastal protection: adapting to climate change and coastal hazards," Ocean \& Coastal Management, vol. 90, pp. 50-57, 2014.

[5] W. N. Adger, T. P. Hughes, C. Folke, S. R. Carpenter, and J. Rockström, "Social-ecological resilience to coastal disasters," Science, vol. 309, no. 5737, pp. 1036-1039, 2005.

[6] CNN. (2018, October 25). Indonesia tsunami: Death toll rises sharply as desperation grows. Available: https://edition.cnn.com/2018/10/02/asia/indonesia-palu-tsunamiearthquake-intl/index.html

[7] M. Besset, E. J. Anthony, P. Dussouillez, and M. Goichot, "The impact of Cyclone Nargis on the Ayeyarwady (Irrawaddy) River delta shoreline and nearshore zone (Myanmar): Towards degraded delta resilience?," Comptes Rendus Géoscience, vol. 349, no. 6, pp. 238-247, 2017.

[8] S. Kottegoda. (2011, March 07). Mainstreaming Gender in Disaster Management Policy: Key issues and Challenges in the Asia-Pacific Region Available: http://www.apwwslwngof.org/index.php?option=com_content\&view=article\&id=88

[9] L. Zou and F. Thomalla, "The Causes of Social Vulnerability to Coastal Hazards in Southeast Asia," in "Stockholm Environment Institute, Working Paper - 2008," Stockholm Environment Institute, Stockholm, Sweden2010, issue Working Paper - 2008.

[10] M. Sjöstedt and M. Povitkina, "Vulnerability of Small Island Developing States to Natural Disasters: How Much Difference Can Effective Governments Make?," The Journal of Environment \& Development, vol. 26, no. 1, pp. 82-105, 2017.

[11] K. C. Seto, M. Fragkias, B. Güneralp, and M. K. Reilly, "A metaanalysis of global urban land expansion," PloS one, vol. 6, no. 8, p. e23777, 2011.

[12] G. Hugo, "Future demographic change and its interactions with migration and climate change," Global Environmental Change, vol. 21, pp. S21-S33, 2011.

[13] International Network for Multi-hazard Early Warning Systems, "Multi-hazard Early Warning Systems: A Checklist," World Meteorological Organization, Geneva, Switzerland2018.

[14] R. Haigh, D. Amartunga, and K. Hemachandra, "A capacity analysis framework for multi-hazard early warning in coastal communities," Procedia engineering, vol. 212, pp. 1139-1146, 2018.

[15] L. Alfieri, P. Salamon, F. Pappenberger, F. Wetterhall, and J. Thielen, "Operational early warning systems for water-related hazards in Europe," Environmental Science \& Policy, vol. 21, pp. 35-49, 2012.
[16] CRED and UNISDR, "Tsunami Disaster Risk: 2016 Past impacts and Projections," 2016.

[17] UN. (2015). Sustainable Development Goals. Available: https://sustainabledevelopment.un.org/?menu=1300

[18] F. Thomalla and R. K. Larsen, "Resilience in the context of tsunami early warning systems and community disaster preparedness in the Indian Ocean region," Environmental Hazards, vol. 9, no. 3, pp. 249$265,2010$.

[19] J. Lauterjung, P. Koltermann, U. Wolf, and J. Sopaheluwakan, "The UNESCO-IOC framework-establishing an international early warning infrastructure in the Indian Ocean region," Natural Hazards and Earth System Sciences, vol. 10, no. 12, pp. 2623-2629, 2010.

[20] UN- ESCAP, "Strengthening regional multi-hazard early warning systems," in "Economic and Social " Economic and Social Commission for Asia and the Pacific Committee on Disaster Risk Reduction2015.

[21] D. Lumbroso, E. Brown, and N. Ranger, "Stakeholders' perceptions of the overall effectiveness of early warning systems and risk assessments for weather-related hazards in Africa, the Caribbean and South Asia," Natural Hazards, vol. 84, no. 3, pp. 2121-2144, 2016.

[22] UN-ESCAP, "Strengthening regional multi-hazard early warning systems " in "Economic and Social Commission for Asia and the Pacific Committee on Disaster Risk Reduction Fourth session " Economic and Social Commission for Asia and the Pacific, Bangkok27-29 October 2015 2015, vol. E/ESCAP/CDR(4)/2

[23] UNESCO-ICO; UN/ISDR/PPEW; WMO, "Assessment of Capacity Building Requirements for an Effective and Durable Tsunami Warning and Mitigation System in the Indian Ocean: Consolidated Report for 16 Countries Affected by the 26 December 2004 Tsunami ", Paris, 2005.

[24] M. Saunders, P. Lewis, and A. Thornhill, Research methods for business students (no. Book, Whole). Harlow: Pearson Education, 2016.

[25] IOC, "Strengthening National Capacities for Tsunami Early Warning and Response Systems in the Indian Ocean. Progress Report as of 25 March 2008," 2008.

[26] R. Basher, "Global early warning systems for natural hazards: systematic and people-centred," Philosophical Transactions of the Royal Society of London A: Mathematical, Physical and Engineering Sciences, vol. 364, no. 1845, pp. 2167-2182, 2006.

[27] F. Thomalla, T. Downing, E. Spanger-Siegfried, G. Han, and J. Rockström, "Reducing hazard vulnerability: towards a common approach between disaster risk reduction and climate adaptation," Disasters, vol. 30, no. 1, pp. 39-48, 2006.

[28] Z. Scott and R.Few, "Strengthening capacities for disaster risk management: Insights from existing research and practice," International Journal of Disaster Risk Reduction, vol. In Press, 2016.

[29] WMO. (2011, 17 February). Good practices for multi-hazard Early Warning Systems (EWS). Available: https://public.wmo.int/en/resources/meteoworld/good-practicesmulti-hazard-early-warning-systems-ews

[30] D. Rogers and V. Tsirkunov, "Implementing hazard early warning systems," Global Facility for Disaster Reduction and Recovery, 2011.

[31] UNISDR, "Sendai Framework for Disaster Risk Reduction 2015 2030," United Nations, Geneva Switzerland2015, Available: http://www.preventionweb.net/files/43291_sendaiframeworkfordrren .pdf, Accessed on: 09032016.

[32] D. S. C. Seng, "Tsunami resilience: Multi-level institutional arrangements, architectures and system of governance for disaster risk preparedness in Indonesia," Environmental science \& policy, vol. 29, pp. 57-70, 2013. 